\title{
Renormalization-Group Transformations and Correlations of Seismicity
}

\author{
Álvaro Corral \\ Departament de Física, Facultat de Ciències, Universitat Autònoma de Barcelona, E-08193 Bellaterra, Barcelona, Spain
}

(Received 25 January 2005; published 7 July 2005)

\begin{abstract}
The effect of transformations analogous to those of the real-space renormalization group are analyzed for the temporal occurrence of earthquakes. A recently reported scaling law for the distribution of recurrence times implies that these distributions must be invariant under such transformations, for which the role of the correlations between the magnitudes and the recurrence times are fundamental. This approach puts the study of the temporal structure of seismicity in the context of critical phenomena.
\end{abstract}

DOI: 10.1103/PhysRevLett.95.028501

The study of the collective properties of earthquakes has a long history, exemplified by the Omori law of aftershocks and the Gutenberg-Richter relation for the number of earthquakes above a given magnitude and, more recently, by the fractal properties of earthquake spatial occurrence [1-4]. Less attention has been paid to the timing of individual earthquakes, for which a unifying picture was missing until the work of Bak et al. [5-7]. The main relevance of that work was the seminal introduction of scaling concepts in statistical seismology, providing a powerful tool to unify descriptions and to derive relations between different quantities [8]. Later, the procedure of Bak et al. was modified to study the distribution of times between consecutive events in any single spatial region with a constant or power-law decaying seismic rate [9-11].

Let us consider the seismicity of an arbitrary spatial region. Given a lower bound $M_{c}$ for the magnitude, and intentionally disregarding the spatial degrees of freedom [12], a marked point process in time of the form $\left(t_{0}, M_{0}\right),\left(t_{1}, M_{1}\right), \ldots$, is obtained, where $t_{i}$ denotes the time of occurrence of event $i$, with a magnitude $M_{i} \geq$ $M_{c}$. The recurrence times are defined as the time intervals between nearest-neighbor (i.e., consecutive) events, $\tau_{i} \equiv$ $t_{i}-t_{i-1}$. In the case of stationary seismicity (characterized by a more or less linear relation between the accumulated number of earthquakes and time [10]), for spatial regions of linear size ranging from $20 \mathrm{~km}$ to the whole world, and for magnitude bounds from 1.5 to 7.5 , the probability densities $D(\tau)$ of the recurrence time were found to verify a universal scaling law [9-11],

$$
D(\tau)=R f(R \tau)
$$

where $f$ is a universal scaling function and the scaling factor $R$ is the rate of seismic occurrence, defined as the mean number of events with $M \geq M_{c}$ in the region, per unit time, and given by the Gutenberg-Richter law, $R\left(M_{c}\right)=R_{0} 10^{-b M_{c}}$, with the $b$ value usually close to 1 and $R_{0}$ depending on the region.

As no separation of mainshocks and aftershocks is performed, the stationary recurrence-time distribution consists of a mixture of different aftershock sequences and more or less independent events; therefore, it is very sur-
PACS numbers: 91.30.Dk, 05.40.-a, 64.60.Ht, 89.75.Da

prising that distinct regions and earthquakes of disparate sizes present such an extreme degree of regularity.

But even more surprising, the scaling relation for the recurrence-time distribution reveals that seismicity is in a highly orchestrated state, in which the removal of events (when the lower bound $M_{c}$ is raised) does not affect the properties of seismic occurrence, as the distribution keeps the same shape (with only a different mean) independently of $M_{c}$. In general, when some events are removed from a point process, the properties of the process do change; therefore, the distribution of earthquake recurrence times constitutes a very special case, invariant under a transformation akin to those of the renormalization group (RG) in real space $[3,13,14]$.

The first step of our renormalization-group transformation consists of the raising of the lower bound $M_{c}$. This implies that only a fraction of events survives the transformation, which leads to a different recurrence-time distribution. (If $M_{c}$ is increased in one unit, we are dealing with an authentic decimation, as only about one-tenth of the events are kept, because of the Gutenberg-Richter law.) The second part of the procedure is the scale transformation, which changes the time scale to make the new system comparable with the original one. A third step, the renormalization of the field $\left(M_{i}\right)$, is not necessary here as we are interested only in the recurrence times.

The Poisson process, characterized by an exponential recurrence-time distribution, represents a trivial solution to this problem when there are no correlations and therefore events are randomly removed. In fact, it has been argued that the scaling function $f$ can be only an exponential function [15]; however, seismic data analysis shows that $f$ clearly departs from an exponential [9], and in this way the relevance of correlations in the structure of seismicity becomes apparent.

The scaling function $f$ is described by a decreasing power law for intermediate times, $R \tau<1$, with an exponent about 0.3 , and by a faster decay for long times, $R \tau>$ 1 , and is well approximated by a gamma distribution [9], except at short times, $R \tau<0.01$, for which the condition of stationarity is usually not fulfilled. Nevertheless, in the nonstationary case the process can be transformed into a 
stationary one with an appropriate nonlinear rescaling of the time axis, and then the same scaling relation is found to hold again [9]. As no model of earthquake occurrence is assumed to obtain these results, they constitute a fundamental characteristic of seismicity.

In general, the time between two consecutive earthquakes, $\tau_{i}$, may depend on the magnitude of the former event, $M_{\text {pre }}=M_{i-1}$, on the previous recurrence time, $\tau_{i-1}$, and also on the occurrence of preceding events, $i-2, i-$ 3 , etc. In their turn, the magnitude of the $i$ th event $M_{i}$ can depend on $\tau_{i}, M_{i-1}, \tau_{i-1}$, and so on. Data analysis suggests that the correlations of the magnitude with previous magnitudes and times can be disregarded [16], whereas the correlations of the recurrence times with history are important $[16,17]$. As a starting point, we consider here only the dependence of $\tau_{i}$ on $M_{i-1}$, ignoring the dependence of $\tau_{i}$ with $\tau_{i-1}$, and the extent of correlations in the past. Note that, although the dependence of the recurrence time with the distance between events can be important, as we are looking at the projection of seismicity in magnitude and time, we do not need to take this effect into account. So, in what follows we study the effect in the structure of seismicity of the simplified case of correlations between the recurrence time and the magnitude of the previous earthquake.

If we raise the magnitude threshold from $M_{c}$ to $M_{c}^{\prime}$ the distribution of recurrence times for events with magnitudes $M \geq M_{c}^{\prime}$ can be obtained from the distribution for events with $M \geq M_{c}$. Assuming that an event with magnitude $M_{0} \geq M_{c}^{\prime}$ has occurred, we can write for the next event above (or at) $M_{c}^{\prime}$,

$$
\begin{aligned}
& P\left[\text { recurrence time }>\tau \text { for events } M \geq M_{c}^{\prime}\right] \\
& \quad=\sum_{j=1}^{\infty} P\left[\tau^{(j)}>\tau \mid M_{1}<M_{c}^{\prime}, \ldots, M_{j-1}<M_{c}^{\prime}, M_{j}\right. \\
& \left.\quad \geq M_{c}^{\prime}\right] P\left[M_{1}<M_{c}^{\prime}\right] \cdots P\left[M_{j-1}<M_{c}^{\prime}\right] P\left[M_{j} \geq M_{c}^{\prime}\right]
\end{aligned}
$$

where $P$ denotes probability, $\mid$ conditional events, and the $j$ th return time is defined, for events with $M \geq M_{c}$, as $\tau_{i}^{(j)}=t_{i}-t_{i-j}$, that is, as the elapsed time between any event and the $j$ th event after it. We have explicitly made use of the fact that in our approach the magnitude is independent of history. Using the Gutenberg-Richter law we define

$$
p \equiv P\left[M \geq M_{c}^{\prime}\right]=10^{-b\left(M_{c}^{\prime}-M_{c}\right)},
$$

and $q \equiv 1-p=P\left[M<M_{c}^{\prime}\right]$, where both probabilities are, in fact, conditioned to $M \geq M_{c}$. Therefore,

$$
\begin{aligned}
& P\left[\text { recurrence time }>\tau \text { for events } M \geq M_{c}^{\prime}\right] \\
& \quad=\sum_{j=1}^{\infty} p q^{j-1} P\left[\tau^{(j)}>\tau \mid M_{1}<M_{c}^{\prime}, \ldots, M_{j-1}<M_{c}^{\prime}, M_{j}\right. \\
& \left.\quad \geq M_{c}^{\prime}\right] .
\end{aligned}
$$

Derivation in this equation with respect to $\tau$ yields the probability densities $D$ of the return times; as the recurrence times are considered independent of each other, we use that the $j$ th-return-time distribution is given by $j$ convolutions of the first-return-time distributions (denoted by the symbol *) to get

$$
\mathrm{T}_{1 / 2} D(\tau)=p D_{\uparrow}(\tau)+q p D_{\uparrow}(\tau) * D_{\downarrow}(\tau)+\cdots
$$

where $T_{1 / 2} D(\tau)$ denotes the probability density for events with $M \geq M_{c}^{\prime}$ as a transformation $\mathrm{T}_{1 / 2}$ of the probability density for events with $M \geq M_{c}, D(\tau)$; more precisely, $D(\tau) \equiv D\left(\tau \mid M \geq M_{c}\right)$, and $\mathrm{T}_{1 / 2} D(\tau) \equiv D\left(\tau \mid M \geq M_{c}^{\prime}\right)$. The subscript $1 / 2$ refers to the fact that this is only the first half of the RG transformation. $D_{\uparrow}$ and $D_{\downarrow}$ denote the recurrence-time probability densities for events above $M_{c}$ conditioned to the fact that the magnitude of the previous event is above or below $M_{c}^{\prime}$ ( $\uparrow$ or $\downarrow$ ), respectively. To be concrete, $\quad D_{\uparrow}(\tau) \equiv D\left(\tau \mid M_{\text {pre }} \geq M_{c}^{\prime}, M \geq M_{c}\right), \quad$ and $D_{\downarrow}(\tau) \equiv D\left(\tau \mid M_{\text {pre }}<M_{c}^{\prime}, M \geq M_{c}\right)$.

It is convenient to look at Eq. (3) in Laplace space, where

$$
\mathrm{T}_{1 / 2} D(s)=p D_{\uparrow}(s)+q p D_{\uparrow}(s) D_{\downarrow}(s)+\cdots .
$$

Notice that we have used the same symbol $D$ both for the probability densities and for their Laplace transforms (which we may call generating functions), although they are different functions, of course. As $q$ and $D_{\downarrow}(s)$ are smaller than 1 (this is general for generating functions), the infinite sum can be performed, turning out that

$$
\mathrm{T}_{1 / 2} D(s)=\frac{p D_{\uparrow}(s)}{1-q D_{\downarrow}(s)}=\frac{p D_{\uparrow}(s)}{1-D(s)+p D_{\uparrow}(s)},
$$

using that $D(\tau)$ is, in fact, a mixture of the distributions $D_{\uparrow}$ and $D_{\downarrow}$, of the form $D=p D_{\uparrow}+q D_{\downarrow}$.

Equation (5) describes the first part of the transformation. The second part is the scale transformation, $T_{2 / 2}$, which puts the distributions corresponding to $M_{c}$ and $M_{c}^{\prime}$ on the same scale. We obtain this by removing the effect of the decreasing of the rate, which, by (2), is proportional to $p$, so

$$
\mathrm{T}_{2 / 2}\left[\mathrm{\top}_{1 / 2} D(\tau)\right]=p^{-1} \mathrm{~T}_{1 / 2} D(\tau / p),
$$

and in Laplace space we get

$$
\mathrm{T}_{2 / 2}\left[\mathrm{~T}_{1 / 2} D(s)\right]=\mathrm{\top}_{1 / 2} D(p s) .
$$

Therefore, the combined effect of both transformations leads to the final expression for the transformation, $T \equiv$ $\mathrm{T}_{2 / 2} \mathrm{~T}_{1 / 2}$,

$$
\top D(s)=\frac{p D_{\uparrow}(p s)}{1-D(p s)+p D_{\uparrow}(p s)} .
$$

The fixed points of the transformation are obtained by the solutions of $T D(s)=D(s)$. This fixed-point equation provides a connection with Eq. (1), both being equivalent. 
In order to get some understanding of the transformation $\mathrm{T}$, we can consider first the case in which there are no correlations between the magnitude and the subsequent recurrence time. Then, $D_{\uparrow}=D_{\downarrow}=D \equiv D_{0}$, and if we introduce $\omega \equiv p s$ and substitute $p=\omega / s$ and $q=1-$ $\omega / s$ in the fixed-point equation corresponding to (8) we get, separating variables and equating to an arbitrary constant $k$ (due to the fact that $p$ and $s$ are independent variables and so are $s$ and $\omega$ )

$$
D_{0}(s)=(1+k s)^{-1},
$$

which is the Laplace transform of an exponential distribution, $D_{0}(\tau)=k^{-1} e^{-\tau / k}$. So, in the case of a process with no correlations, the only scale invariant distribution is, as one could have expected, the exponential, characteristic of the Poisson process.

Correlations introduce new functions in the process. In our case, in order to iterate the transformation $T$ we need to know how $D_{\uparrow}$ transforms as well. It turns out that $D_{\uparrow}$ verifies an equation very similar to Eq. (8), which depends on $D_{\Uparrow}(\tau) \equiv D\left(\tau \mid M_{\text {pre }} \geq M_{c}^{\prime \prime}, M \geq M_{c}\right)$. So, in order to apply $\mathrm{T}$ again, we also need an equation to transform $D_{\Uparrow}$, which in turn depends on higher values of the magnitude threshold. In this way we obtain a hierarchy of equations. An easy way to break this hierarchy is to assume that, at least at the fixed point, $D_{\uparrow}$ has the same functional form as $D$, but in a different scale. So, let us assume, as a first approximation, the following ansatz:

$$
D_{\uparrow}(\tau) \simeq \Lambda D(\Lambda \tau)
$$

where $\Lambda$ depends on $M_{c}^{\prime}-M_{c}$ with $\Lambda(0)=1$.

This ansatz (10) can be justified with real data. Figure 1 illustrates this, using worldwide earthquakes from the NEIC PDE catalog [9]. The distributions $D_{\uparrow}$ for different values of $M_{c}^{\prime}$ keeping $M_{c}=6$ collapse onto a single curve under rescaling of the axes. For each distribution the scaling factor is the inverse of its mean value, $R_{\uparrow}$, and therefore $\Lambda=R_{\uparrow} / R$. [Nevertheless, Eq. (10) only seems to be valid at not very large $M_{c}^{\prime}-M_{c}$; see Ref. [16].] The behavior of $\Lambda$ as a function of $M_{c}^{\prime}$ appears in the inset of Fig. 1. A horizontal line would indicate the absence of correlations, as $R_{\uparrow}$ would be identical to $R$ and therefore $D_{\uparrow}=D$. As $R_{\uparrow}$ increases with the magnitude of the previous event, this means that the mean time between events decreases, leading to increasing clustering after larger events.

In the figure, fits of the type $\Lambda\left(M_{c}^{\prime}-M_{c}\right)=$ $A+C\left(M_{c}^{\prime}-M_{c}\right)$ and $\Lambda\left(M_{c}^{\prime}-M_{c}\right)=A e^{C\left(M_{c}^{\prime}-M_{c}\right)}$ are shown; in both cases it turns out that $A \simeq 1$ and $C$ is in the range $0.18-0.20$. (Note that this behavior of $\Lambda$ does not strictly verify Båth's law, which would imply $\Lambda=1$ for $M_{c}^{\prime}-M_{c}<1.2$ [18].)

Returning to our calculation, in Laplace space $D_{\uparrow}(s) \simeq$ $D(s / \Lambda)$; therefore, the transformation (8) turns out to be

$$
\mathrm{T} D(s) \simeq \frac{p D(p s / \Lambda)}{1-D(p s)+p D(p s / \Lambda)} .
$$

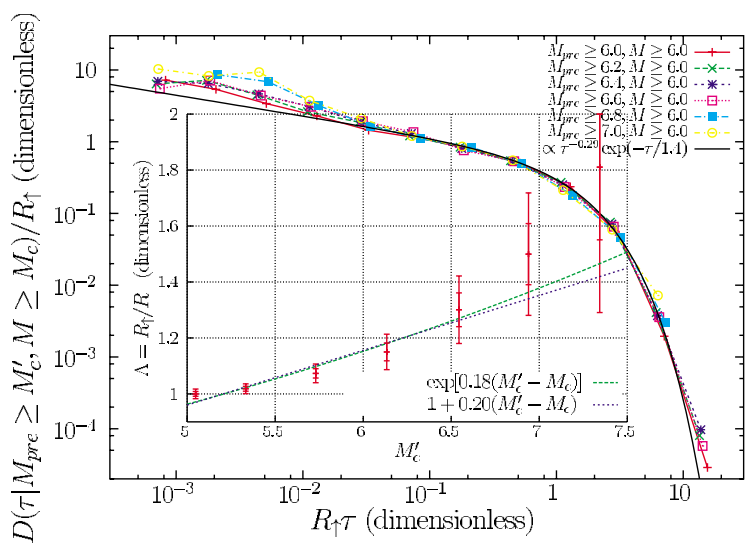

FIG. 1 (color online). Analysis of worldwide earthquakes from 1973 to 2002. Main: recurrence-time probability densities conditioned to $M_{\text {pre }} \geq M_{c}^{\prime}, M_{c}^{\prime}$ varying from 6 to 7 and with $M \geq 6$. The data collapse indicates that $D_{\uparrow}(\tau)$ may verify the same scaling relation as $D(\tau)$. The solid line is a fit to $D(\tau)$, turning out to be $\propto e^{-\tau / 1.4} / \tau^{0.29}$. Inset: inverse of the mean recurrence time, $R_{\uparrow}$, scaled by $R$ for recurrence periods started by events with $M_{\text {pre }} \geq M_{c}^{\prime}$ and ending with $M \geq 5$. The error bars mark 2 standard deviations of the mean value, and the two curves are the linear and exponential fits explained in the text, which do not take into account the last two points.

As this discrete transformation is difficult to deal with, we look at the infinitesimal transformation defined by $M_{c}^{\prime} \rightarrow M_{c}$. Introducing $\delta \equiv M_{c}^{\prime}-M_{c}$, this implies $p=$ $10^{-b\left(M_{c}^{\prime}-M_{c}\right)} \simeq 1-B \delta$ with $B=b \ln 10, D(p s)=D(s)-$ $B s D^{\prime}(s) \delta, \quad \Lambda \simeq 1+C \delta$, and $D(p s / \Lambda)=D(s)-(B+$ $C) s D^{\prime}(s) \delta$. Substituting in Eq. (11) and up to first order in $\delta$ we get

$$
\begin{aligned}
\top D(s) \simeq & D(s)+\left\{[D(s)-1]\left[B D(s)+C s D^{\prime}(s)\right]\right. \\
& \left.-B s D^{\prime}(s)\right\} \delta .
\end{aligned}
$$

In order to find the fixed point of this transformation, we impose that the coefficient of $\delta$ is zero, obtaining

$$
D^{\prime}(s)=-\frac{B D(s)[1-D(s)]}{s\{B+C[1-D(s)]\}},
$$

whose direct integration is easy, yielding

$$
k s D^{1+\varepsilon}(s)+D(s)-1=0,
$$

where the exponent $1+\varepsilon$ comes from the definition $\varepsilon \equiv$ $C / B$. We immediately see that in the case of no correlations, $C=0$, one has $\varepsilon=0$, recovering Eq. (9) and then the exponential form for $D(\tau)$.

Although there are a few values of $\varepsilon$ for which Eq. (14) can easily be solved $(\varepsilon=-1 / 2)$ and even transformed back in Laplace $(\varepsilon=1)$, we are more interested in general properties. Let us study first the case $\varepsilon \rightarrow 0$, characteristic of weak correlations, $C \simeq 0$. We can write the solution $D(s)$ as a perturbation of the Poisson behavior corresponding to $\varepsilon=0$, i.e., $D(s)=D_{0}(s)+\varepsilon u(s)+O\left(\varepsilon^{2}\right)$, with $D_{0}(s)=$ $(1+k s)^{-1}$; see Eq. (9). Substituting into Eq. (14), we get 


$$
u(s)=k s(1+k s)^{-2} \ln (1+k s),
$$

and inverting in Laplace $u(s)$ and $D_{0}(s)$, we obtain [19]

$D(\tau)=\left\{1+\varepsilon\left[\left(\ln \frac{\tau}{k}+\gamma\right)\left(\frac{\tau}{k}-1\right)-\frac{\tau}{k}\right]\right\} \frac{e^{-\tau / k}}{k}+O(\varepsilon)^{2}$,

where $\gamma$ in this case is Euler's constant, $\gamma \equiv-\Gamma^{\prime}(1)$.

We may derive an equivalent expression for $D(\tau)$, up to first order in $\varepsilon$, using the relation $(k / \tau)^{\varepsilon} \simeq 1-\varepsilon \ln (\tau / k)$ and $\Gamma(1-\varepsilon) \simeq 1+\gamma \varepsilon$; so

$$
D(\tau) \simeq\left(\frac{k}{\tau}\right)^{\varepsilon} \frac{e^{-\tau / k}}{k \Gamma(1-\varepsilon)}\left[1-\frac{1}{1-\varepsilon}\left(\frac{\tau}{k}\right)+\Gamma(1-\varepsilon)\left(\frac{\tau}{k}\right)^{1+\varepsilon}\right],
$$

which is not a systematic expansion in $\varepsilon$ but shows that $D(\tau)$ can be approximated by a gamma distribution in a certain domain, in agreement with observational-data analysis [9].

This behavior is by no means exclusive of $\varepsilon \simeq 0$. If in Eq. (14) we consider the limit $s \rightarrow \infty$ we get, as $D(s) \rightarrow 0$ (which is general for generating functions),

$$
D(s) \rightarrow 1 /(k s)^{1 / 1+\varepsilon},
$$

and, if $1+\varepsilon>0$, by means of a Tauberian theorem [20],

$$
D(\tau) \rightarrow \frac{1}{k \Gamma(1-\zeta)}\left(\frac{k}{\tau}\right)^{\zeta} \text { for } \tau \rightarrow 0
$$

with $\zeta=\varepsilon /(1+\varepsilon)$. This implies that for all $\varepsilon>0$, which corresponds to $\Lambda>1$, and then to a shortening of the recurrence times after large earthquakes, we get a decreasing power law, which is a signature of clustering, and in concordance with real data [9]. (The situation would be reversed if $-1<\varepsilon<0$.)

Further insight can be obtained from Eq. (14), writing it as $D=1-k s D^{1+\varepsilon}$, which can be iterated to get the Taylor expansion of $D(s)$. Up to second order, it turns out that $D(s) \simeq 1-k s+(1+\varepsilon) k^{2} s^{2}$, and as the coefficients of $s^{n}$ yield the moments of the distribution (with a $(-1)^{n} / n$ ! factor [20]) we conclude that $\langle\tau\rangle=k,\left\langle\tau^{2}\right\rangle=$ $2(1+\varepsilon) k^{2}$ and the coefficient of variation is $c v \equiv$ $\sqrt{\left\langle\tau^{2}\right\rangle-\langle\tau\rangle^{2}} /\langle\tau\rangle=\sqrt{1+2 \varepsilon}$. In fact, the Taylor expansion can be obtained up to any order, and so all the moments $\left\langle\tau^{n}\right\rangle$ exist and $D(\tau)$ decays faster than any power law for $\tau \rightarrow \infty$, in agreement again with the observations [9].

Nevertheless, this agreement becomes a disagreement when made quantitative. Which may be the reason of the disagreement? In fact, one can verify that the solutions that we find for Eq. (13) are not fixed points of the transformation (8). The reason is the approximation given by Eq. (10), which, in addition, does not seem to be experimentally valid for $M_{c}^{\prime}-M_{c}>1$. So we may obtain functions very close to the true fixed points of Eq. (8), but after repeated application of the RG transformation these functions flow towards the true solution. Molchan has shown that these fixed points can only be exponential [21]. We believe the inclusion of stronger correlations in the model will yield a better agreement between theory and observations.

To summarize, our approach mainly consists of a simplification of real seismicity, which allows one to understand the complex structure of seismic occurrence in the time and magnitude domains. Simply by imposing the selfsimilarity of the process, a general characterization of the recurrence-time distribution can be obtained. With this study we have shown how the structure of seismic occurrence in time and magnitude can be understood as a critical phenomenon and then constitutes a statistical-physics problem [22].

Y. Y. Kagan, G. Molchan, M. Paczuski, D. L. Turcotte, the Ramón y Cajal program, and Projects No. BFM200306033 and No. 2001-SGR-00186 are acknowledged.

[1] T. Utsu, in International Handbook of Earthquake and Engineering Seismology, edited by W.H.K. Lee, H. Kanamori, P. C. Jennings, and C. Kisslinger (Academic Press, New York, 2002), Pt. A, p. 719.

[2] Y. Y. Kagan, Physica (Amsterdam) 77D, 160 (1994).

[3] D. L. Turcotte, Fractals and Chaos in Geology and Geophysics (Cambridge University Press, Cambridge, 1997), 2nd ed.

[4] D. L. Turcotte and B.D. Malamud, in International Handbook of Earthquake and Engineering Seismology (Ref. [1]), Pt. A, p. 209.

[5] P. Bak, K. Christensen, L. Danon, and T. Scanlon, Phys. Rev. Lett. 88, 178501 (2002).

[6] A. Corral, Phys. Rev. E 68, 035102(R) (2003).

[7] A. Corral, Physica (Amsterdam) 340A, 590 (2004).

[8] As we will see, by scaling we do not mean just power-law relations, as it is sometimes assumed.

[9] A. Corral, Phys. Rev. Lett. 92, 108501 (2004).

[10] A. Corral, Nonlinear Process. Geophys. 12, 89 (2005).

[11] A. Corral, Phys. Rev. E 71, 017101 (2005).

[12] Of course, the position of the events is important; see J. Davidsen and M. Paczuski, Phys. Rev. Lett. 94, 048501 (2005).

[13] L. P. Kadanoff, Statistical Physics: Statics, Dynamics and Renormalization (World Scientific, Singapore, 2000).

[14] K. Christensen and N.R. Moloney, Complexity and Criticality (Imperial College Press, London, 2005).

[15] G. Molchan, Pure Appl. Geophys. 162, 1135 (2005).

[16] A. Corral, Phys. Rev. Lett. (to be published).

[17] V. Livina, S. Tuzov, S. Havlin, and A. Bunde, Physica (Amsterdam) 348A, 591 (2005).

[18] D. L. Turcotte (private communication).

[19] Handbook of Mathematical Functions, edited by M. Abramowitz and I. A. Stegun (Dover, New York, 1965).

[20] W. Feller, An Introduction to Probability Theory and Its Applications (Wiley, New York, 1971), Vol. 2, 2nd ed.

[21] G. Molchan (private communication).

[22] P. Bak, How Nature Works: The Science of Self-Organized Criticality (Copernicus, New York, 1996). 